\title{
QUEEN'S
UNIVERSITY
BELFAST
}

\section{Ion acceleration in electrostatic field of charged cavity created by ultra-short laser pulses of $1020-1021 \mathrm{~W} / \mathrm{cm} 2$}

Bychenkov, V. Y., Singh, P. K., Ahmed, H., Kakolee, K. F., Scullion, C., Jeong, T. W., Hadjisolomou, P., Alejo, A., Kar, S., Borghesi, M., \& Ter-Avetisyan, S. (2017). Ion acceleration in electrostatic field of charged cavity created by ultra-short laser pulses of 1020-1021 W/cm2. Physics of Plasmas, 24(1), [ 010704 ]. https://doi.org/10.1063/1.4975082

Published in:

Physics of Plasmas

Document Version:

Peer reviewed version

Queen's University Belfast - Research Portal:

Link to publication record in Queen's University Belfast Research Portal

Publisher rights

Copyright 2017 AIP Publishing

This article may be downloaded for personal use only. Any other use requires prior permission of the author and AIP Publishing.

\section{General rights}

Copyright for the publications made accessible via the Queen's University Belfast Research Portal is retained by the author(s) and / or other copyright owners and it is a condition of accessing these publications that users recognise and abide by the legal requirements associated with these rights.

Take down policy

The Research Portal is Queen's institutional repository that provides access to Queen's research output. Every effort has been made to ensure that content in the Research Portal does not infringe any person's rights, or applicable UK laws. If you discover content in the Research Portal that you believe breaches copyright or violates any law, please contact openaccess@qub.ac.uk. 


\title{
Ion acceleration in electrostatic field of charged cavity created by ultra-short laser pulses of
} $10^{20}-10^{21} \mathrm{~W} / \mathrm{cm}^{2}$

\author{
V. Yu. Bychenkov ${ }^{1,2}$, P. K. Singh ${ }^{3}$, H. Ahmed ${ }^{4}$, K. F. Kakolee ${ }^{3}$, C. Scullion ${ }^{4}$, T. W. Jeong ${ }^{3,5}$, \\ P. Hadjisolomou ${ }^{4}$, A. Alejo ${ }^{4}, \mathrm{~S} . \mathrm{Kar}^{4}, \mathrm{M}$. Borghesi ${ }^{4}$, and S. Ter-Avetisyan ${ }^{3,5}$ \\ ${ }^{I}$ P. N. Lebedev Physics Institute, Russian Academy of Sciences, Moscow 119991, Russia \\ ${ }^{2}$ Center for Fundamental \& Applied Research, VNIIA, ROSATOM, Moscow 127055, Russia \\ ${ }^{3}$ Center for Relativistic Laser Science, Institute of Basic Science (IBS), Gwangju 61005, Korea \\ ${ }^{4}$ School of Mathematics and Physics, The Queen's University of Belfast, Belfast, BT7 1NN, UK \\ ${ }^{5}$ Department of Physics and Photon Science, Gwangju Institute of Science and Technology (GIST), \\ Gwangju 61005, Korea
}

Ion acceleration resulting from the interaction of ultra-high intensity and ultra-high contrast $\left(\sim 10^{10}\right)$ laser pulses with thin $\mathrm{Al}$ foil targets at $30^{\circ}$ angle of laser incidence is studied. Proton maximum energies of 30 and $18 \mathrm{MeV}$ are measured along the target normal rear and front sides, respectively, showing intensity scaling as $I^{b}$. For the target front $b_{\text {front }}=0.5-0.6$ and for the target rear $b_{\text {rear }}=0.7-0.8$, for the intensity range $10^{20}-10^{21} \mathrm{~W} / \mathrm{cm}^{2}$. The fast scaling from the target rear $\sim I^{0.75}$ can be attributed to the enhancement of laser energy absorption as already observed at relatively low intensities. The backwards acceleration of the front side protons with intensity scaling as $\sim I^{0.5}$ can be attributed to the to the formation of a positively charged cavity at the target front via ponderomotive displacement of the target electrons at the interaction of relativistic intense laser pulses with solid target. The experimental results are in a good agreement with theoretical predictions.

The acceleration of ions to multi-MeV energies from foil targets has been investigated extensively, over the last decade using intense laser pulses $\left(10^{18}-10^{20} \mathrm{~W} / \mathrm{cm}^{2}\right)$. The mechanisms of ion acceleration have been reviewed in Ref. [1] and the perspectives of this research have been inferred. Understanding and controlling the acceleration mechanisms, determining the relevant energy scaling laws and efficiency of the acceleration processes are key steps in view of source development for potential applications. The fast developing laser technology enables access to unprecedented intensities (above $10^{21} \mathrm{~W} / \mathrm{cm}^{2}$ ) with remarkably improved pulse temporal contrast by employing several techniques such as cross-polarized wave [2], plasma mirrors [3] and saturable absorbers [4]. In this new intensity regime the laser plasma interaction conditions are changing significantly and experiments aiming not only to obtain/confirm the scaling laws, but also to test the different acceleration mechanisms active under these new conditions are essential.

The generally accepted scenario of ion acceleration employing ultrashort laser pulses is that the ions gain their energy in a strong quasistatic electric field arising from spatial charge separation due to the displacement of the fast electrons created by the laser field. In particular for targets much thicker than the laser penetration length, this scenario leads to the so-called target normal sheath acceleration (TNSA) mechanism [5]. The energy scaling of forward accelerated protons with $\sim 50$ fs lasers at intensities ranging from $\sim 10^{18}$ to $10^{19} \mathrm{~W} / \mathrm{cm}^{2}$ and modest intensity contrast ratios has been reviewed in $[6,7]$. At much higher laser intensities $\left(\sim 10^{21} \mathrm{~W} / \mathrm{cm}^{2}\right)$ and improved pulse 
Publishippgt ast, the generation of high-energy protons is likely to follow different acceleration scenarios and energy scaling laws due to the formation of a relativistic skin depth at the target front, arising from relativistic self-induced transparency, which may have influence also on the acceleration of ions at the target rear.

Experiments, where proton emission was measured along both rear surface and front surface target normal directions, employing ultra-high laser pulse contrast $\left(10^{10}\right)$ at intensity $10^{18} \mathrm{~W} / \mathrm{cm}^{2}$, have shown almost identical proton energies for target thicknesses from $0.1 \mu \mathrm{m}$ to $100 \mu \mathrm{m}$ [8] with maximum proton energies up to $5 \mathrm{MeV}$. It was confirmed in these experiments that the maximum proton energies are proportional to the $p$ component of the laser electric field only and not to the ponderomotive force. More recently, similar experiments irradiating 0.015 $90 \mu \mathrm{m}$ foil targets at an intensity $\leq 10^{20} \mathrm{~W} / \mathrm{cm}^{2}$ have also demonstrated an almost symmetric behavior for protons accelerated from rear and front target surfaces [9] with maximum proton energies of $\sim 10-12 \mathrm{MeV}$. Those experiments covering the intensity range $10^{18} \mathrm{~W} / \mathrm{cm}^{2}-10^{20} \mathrm{~W} / \mathrm{cm}^{2}[8,9]$ were interpreted on the basis that the same TNSA concept of ion acceleration applied to both target surfaces. However, in Ref. [10], at laser intensity $2 \times 10^{20}$ $\mathrm{W} / \mathrm{cm}^{2}$ and ultra-high pulse contrast, a strong imbalance between forward and backward proton acceleration was observed for targets thicknesses from 0.05 up to $10 \mu \mathrm{m}$. A maximum proton energy of about $12 \mathrm{MeV}$ was measured along the rear surface target normal direction, while from the front surface the energies hardly reached $1 \mathrm{MeV}$. Such a strong asymmetry in proton energies from target rear and front surfaces may be connected to particular conditions created at the target front during the laser intensity increase affecting significantly acceleration at the target front while at the target rear the "standard" TNSA is virtually unaffected.

Here, we discuss ion acceleration resulting from the interaction of even higher intensities (above $10^{21} \mathrm{~W} / \mathrm{cm}^{2}$ ) and ultra-high contrast $\left(10^{10}\right)$ laser pulses with a foil target, paying particular attention to the accelerated ions' energy scaling with laser intensity. Under this unexplored intensity regime, the Coulomb field of the charged nonlinear relativistic skin layer is likely to play an important role in the acceleration of ions from the target front. The TNSA scenario [11, 12], which might be applicable to the backward front side proton acceleration occurs in an adiabatic plasma expansion regime [13] when the laser pulse terminates. In this regime the electrons are cooled down rapidly and therefore the transformed energy to protons is not as effective as it can be in an isothermal regime at the target rear [14]. This is why it is unlikely that the front side TNSA proton energy can be comparable to the energy of protons from rear side TNSA at the intensities discussed here.

Experiments in the intensity regime of $10^{20}-10^{21} \mathrm{~W} / \mathrm{cm}^{2}$ were performed on the Ti:Sa Petawatt laser system at the Center for Relativistic Laser Science (CoReLS), IBS, Gwangju [15] where particular attention was devoted to establishing energy scaling laws for the accelerated ions emitted along the normal direction to both front and rear surfaces. Ion acceleration was monitored in both directions by varying laser intensity. The accelerated protons were measured from an optimised target position relative to laser focus, chosen in order to achieve the maximum possible proton energy for every target thickness.

schematic of the experimental set up is shown in Fig 1. A p-polarized, $30 \mathrm{fs}$ laser pulse, having central wavelength of $800 \mathrm{~nm}$ was focused using an $\mathrm{f} / 3$ gold-coated off-axis parabolic mirror on $6 \mu \mathrm{m}$ thick $\mathrm{Al}$ foil targets at an angle of incidence $30^{\circ}$. The focal spot, measured with attenuated laser energy, had nearly $30 \%$ of energy confined in the $4 \mu \mathrm{m}$ FWHM. The temporal contrast of the laser pulse was characterized by a scanning third order 
correlator with a dynamic range of $10^{10}$. Employing technique of "saturable absorber" laser pulse temporal contrast $\sim 5 \times 10^{-10}$ was achieved in a few ps before main pulse. Additionally, the laser pulse contrast was confirmed to be high by measuring the proton energies from the thin foil targets: $0.4,0.2$ and $0.1 \mu \mathrm{m}$. While changing laser intensity from $2 \times 10^{20} \mathrm{~W} / \mathrm{cm}^{2}$ up to $1.2 \times 10^{21} \mathrm{~W} / \mathrm{cm}^{2}$ the observed continuous proton energy increase from the targets front and rear sides suggests that there is no disruption in the interaction conditions in this large intensity range, which may occur if the pre-pulse level at higher intensities will become significantly high to generate preplasma. The latter is expected to make a noticeable change in the interaction condition, which will result in an observable change in the proton energy scaling when laser intensity is increased.

To position the thin foil in the laser focal plane a target position monitoring system was used with accuracy of few micrometers, as described in [16]. Two Thomson parabola spectrometers coupled to absolutely calibrated microchannel plate (MCP) detectors [17] were employed to record the energy spectra of accelerated ions along the rear surface and front surface target normal directions. The solid angle for ion collection was $4.7 \times 10^{-9} \mathrm{sr}$ in both the spectrometers. The recorded, parabolic ion traces were analyzed using a MATLAB code. Fig. 2 show the raw MCP images obtained following the interaction of $8.0 \times 10^{20} \mathrm{~W} / \mathrm{cm}^{2}$ (Fig. 2(a) and 2(d)) and $2.5 \times 10^{20} \mathrm{~W} / \mathrm{cm}^{2}$ (Fig. 2(b) and 2(e)) intense laser pulse $\left(10^{21} \mathrm{~W} / \mathrm{cm}^{2}\right.$, maximum intensity achieved in the experiment) with the $6 \mu \mathrm{m} \mathrm{Al}$ foils, by employing Thomson spectrometers along the rear and front target normal directions respectively. The raw images highlight the acceleration of multi-species ions in both directions. The proton energy distributions corresponding to the ion traces presented in the Fig.2 (a), (b) and (d), (e) are shown in the Fig. 2 (c) and (f), correspondingly. Cut-off energies of $30 \mathrm{MeV}$ and $18 \mathrm{MeV}$ for protons from the target rear and front surfaces were measured respectively.

The intensity on target was varied throughout the experiment by changing the laser energy. The variation of the maximum proton energies observed, with respect to laser intensity, in the forward and backwards directions, is shown in Fig. 3. As expected, the proton energy increases with incident laser intensity, reaching a maximum of $\sim 30$ $\mathrm{MeV}$ (from target rear surface), at an intensity of $\left(0.8 \times 10^{21} \mathrm{~W} / \mathrm{cm}^{2}\right)$. The proton energy scales with laser intensity as $\varepsilon \propto I^{b}$, where at the target front $b_{\text {front }}=0.55 \pm 0.05$ and at the target rear $b_{\text {rear }}=0.75 \pm 0.05$ in the $10^{20}-10^{21}$ $\mathrm{W} / \mathrm{cm}^{2}$ intensity range. The difference in the scaling for the protons observed along the two directions suggests that different mechanisms might be involved in the acceleration of ion beams from the two surfaces.

The fast scaling from the target rear $\sim I^{0.75}$ (i.e. faster than the standard TNSA $\sim I^{0.5}$ dependence associated with the ponderomotive scaling [1]) is similar to that what was observed in the [7] at lower laser intensities and predicted by the numerical simulations in [18], where this effect can be attributed to the enhancement of laser absorption with the intensity increase. Forward acceleration of the protons follows a fast-scaling TNSA scenario, as already observed in experiments at relatively low intensities.

The measured maximum proton energies at short laser pulse $(\tau<60 f s)$ interaction with thin foils $(l>$ $0.1 \mu \mathrm{m}$ ), where TNSA-like acceleration scheme is applicable, as a function of laser intensity along the target forward and backward direction is shown in Fig. 4(a) and 4(b), respectively. The tendency of proton energy increase with increasing laser intensity is apparent and it increases much faster at high intensities than the widely accepted TNSA dependence $\sqrt{I}$ for both from front and rear side of the target. As a whole the presented results confirm the possibility of achieving even high energies employing TNSA scheme. The data for forward accelerated protons 
from the literature (Fig. 4(a)) seems to be showing a trend consistent with the measurements in present study. However for the backward accelerated protons (Fig. 4(b)) not many data points are available, indicating the requirements from the laser conditions of high contrast and intensity to accelerate backward protons to 10 's MeV energy.

Experimentally measured backward accelerated protons energy scaling can be understood in the scenario where interaction of high contrast and relativistic laser pulse with solid density plasma creates a charged cavity by the laser ponderomotive force at the target front which pushes the electrons inside the target. On the basis of this idea, we propose a qualitative theoretical model of backward proton acceleration during the interaction of high contrast PW pulses with solid targets. The measurements of the backwards accelerated ions are broadly consistent with the proposed scenario.

A simple model for the penetration of a highly relativistic $(a>1)$ laser pulse into an overdense plasma is based on the formation of a relativistic skin depth $l_{s k} \sim a\left(c / \omega_{p e}\right)$, where $\omega_{p e}$ is the electron plasma frequency, $a$ is the normalized laser field amplitude, and $c$ is the speed of light. In such concept the effect of plasma Coulomb field in self-induced transparency is ignored, which instead became recently a topic of intense research [19-23]. The ponderomotive force pushes the electrons deep into the target in the form of a moving electron density spike and produces a charge separation layer (a cavity in a realístic 3D geometry) extending from the target's edge to the laser pulse front. The transverse ponderomotive force of the laser pulse isolates the charged cavity from the surrounding plasma similar to what happens in a hole-boring scenario [5]. The electron spike experiences a strong restoring electrostatic field due to the charged layer left behind, unless a balance between the Coulomb force and the ponderomotive force is achieved. Such balance gives a rough estimate of the nonlinear relativistic skin depth [24], $l_{s k}^{N L}$, as

$$
l_{s k}^{N L} \sim a \frac{c}{\omega} \frac{n_{c}}{n_{e}},
$$

which is roughly the optimum foil thickness to produce ions with maximum energy [18]. In Eq. (1) $n_{e}$ is the electron plasma density and $n_{c}$ is the electron critical density.

In a steady-state model for a circularly polarized laser pulse in the relativistic cold-fluid approximation [19, 22] the total charge of the electron spike $Q_{e}^{(s)}$ is equal to the total ion charge in the cavity of depth $l_{s k}^{N L}$. As a result, the electrostatic field near the plasma front side, shown schematically in Fig. 5(a), prevents backward ion acceleration and only forward acceleration occurs. This process was proposed as a mechanism of front side forward ion acceleration [22]. Obviously, this idealized scheme of total charge compensation $Q_{e}^{(s)}=Q_{i}$ does not apply to the case of linearly polarized light pulse which intensively heats the electrons so that they can partly leave the interaction area, leading to $Q_{e}^{(s)}<Q_{i}$. The electrostatic field resulting in this situation is schematically shown in Fig. 5(b), and may now accelerate ions in the backward direction. Note, that even for circularly polarized pulses an electric field profile as shown in Fig. 5(b) is typical for a wide range of laser-plasma parameters (e.g. Fig. 9 in Ref. [23]) and clearly differs from the predictions of cold fluid theory.

Let us consider the Coulomb expansion of a positively charged layer of length $l_{s k}^{N L}$ with light ions (protons) distributed as an impurity near the plasma-vacuum boundary. The impurity will be accelerated from the target 
Publishimugace by the electrostatic field $E=4 \pi q_{+} l_{s k}^{N L}$, where $q_{+}$is the charge density of the nonlinear skin layer. If the inner side of the charged layer within the target coincides with the electron spike with total charge $Q_{e}^{(s)}$, this field will be reduced by $\left(1-Q_{e}^{(s)} / Q_{i}\right)$ times and therefore:

$$
E=4 \pi q_{+} l_{s k}^{N L}\left(1-\frac{Q_{e}^{(S)}}{Q_{i}}\right)
$$

A proton in the field of Eq. (2) gains an energy, $\varepsilon$, at a distance equal to the radius, $R$, of the laser focal spot, given by

$$
\varepsilon=4 \pi e q_{+} l_{s k}^{N L} R\left(1-\frac{Q_{e}^{(S)}}{Q_{i}}\right)
$$

The charge density $q_{+}$within the charged cavity is also partly neutralized by electrons diffusing from the electron spike or entering the cavity from the transverse directions. Correspondingly, $q_{+}=Z_{e n} n_{i 0}\left(1-Q_{e}^{(c)} / Q_{i}\right)$, or $q_{+}=$ $e n_{e 0}\left(1-Q_{e}^{(c)} / Q_{i}\right)$ where $Z e$ is the ion charge, $n_{e(i) 0}$ is the electron (ion) target density, and $Q_{e}^{(c)}$ is the residual total electron charge in the cavity.

By using Eq. (1), we rewrite Eq. (3) for the proton cut off energy in the following form:

$$
\varepsilon \simeq a \frac{\omega R}{c}\left(1-\frac{Q_{e}^{(S)}}{Q_{i}}\right)\left(1-\frac{Q_{e}^{(c)}}{Q_{i}}\right) m_{e} c^{2} \propto \sqrt{I}
$$

where $a=0.85 \lambda[\mu \mathrm{m}] \sqrt{I\left[10^{18} \mathrm{~W} / \mathrm{cm}^{2}\right]}, \lambda=2 \pi \mathrm{c} / \omega$ is the laser wavelength, and $I$ is the laser intensity. The estimate of Eq. (4) shows that maximum proton energy does not depend on the laser wavelength and has a square root scaling with laser intensity. Standard TNSA results in $\varepsilon \propto T_{h}$, where $T_{h}$ is the typical hot electron energy ("temperature"). For the ponderomotive scaling [5]: $T_{h} \propto \sqrt{I_{a b s}}$, where the absorbed laser intensity may itself nonlinearly depend on $I$ through the absorption coefficient, $A(I)$, i.e. $I_{a b s}=A(I) \times I$ (e.g. [18] ). Since for TNSA protons $\varepsilon \propto T_{h}$, the intensity dependence of cavity accelerated protons is expected to be weaker than for TNSA if the absorption coefficient increases with intensity $(1)$.

The estimate in Eq. (4) does not account for a possible electron cloud near the target front side due to the effect of vacuum heating, i.e., so called $[V \times B]$ [25] or/and Brunel [26] electrons. This is why Eq. (4) may somewhat overestimate the maximum proton energy. However, if the vacuum electron density is not large, $<<a n_{c}(1-$ $\left.Q_{e}^{(S)} / Q_{i}\right)\left(1-Q_{e}^{(c)} / Q_{i}\right)$, the estimate (4) should hold.

The effects of electron spike charge loss and cavity charge neutralization $\left(Q_{e}^{S, c}\right)$ are very important. They depend on the laser pulse temporal and radial shape and should be studied in detail using multi-dimensional PIC simulations. As an example, if assume approximately the same $\sim 70 \%$ neutralization for both spike and cavity by electrons, $Q_{e}^{(c)} \sim Q_{e}^{(c)} \sim 0.7 Q_{i}$, one obtains from Eq. (4) $\varepsilon \sim 18 \mathrm{MeV}$ for $I \sim 1.4 \times 10^{21} \mathrm{~W} / \mathrm{cm}^{2}$ and $R=2 \mu \mathrm{m}$

According to the model discussed above, a field distribution as shown in Fig. 5(b) is sustained at the target front surface as long as the laser pulse irradiates the target. Therefore, a "standard" TNSA expansion is constrained at the front surface within the pulse duration- while it applies to the ion acceleration from the target rear. Standard TNSA expansion from the front can only start after the end of the pulse - at this stage however ion acceleration will be 
Publishiingf ective due to the rapid cooling of electrons in the adiabatic plasma expansion [13], with the electron energy decreasing rapidly as $\left(\omega_{p i} t\right)^{-2}[13]$.

An estimate of hot electron density from the balance of energy fluxes gives: $n_{h}=a n_{c} / \sqrt{2}$, that allows to estimate $\omega_{p i}$ (ion Langmuir frequency) and the energy of TNSA protons from the target rear using $\varepsilon=0.35 \operatorname{aln}^{2}(a)$ from [27], which extends the TNSA model [14] for two electron temperatures plasma: laser generated hot electrons and cold target electrons [28]. For absorption $A=25 \%$ and $I \sim 1.4 \times 10^{21} \mathrm{~W} / \mathrm{cm}^{2}$ the estimate gives about $30 \mathrm{MeV}$. If one assumes a weak absorption dependence, as in Ref. [18]: $A \sim I^{0.2}$, the ion energy scaling from [14] becomes $I^{0.75}$. On the other end, the slower scaling observed at the target front, as compare to the target rear, is broadly consistent with the predictions of equation (4) and with the charged cavity acceleration model presented earlier.

To summarize, ion acceleration experiments carried out at intensities $10^{20}-10^{21} \mathrm{~W} / \mathrm{cm}^{2}$ and very high temporal contrast, have shown a clear asymmetry in energy scaling for protons accelerated along the target normal in the forward and backwards direction. To explain this difference, we propose a new model of backward ion acceleration in the Coulomb field of the charged nonlinear relativistic skin layer created by the interaction of Petawatt laser pulses with solid target. During the interaction, at the front surface of the target, a charged cavity is produced by laser ponderomotive force pushing the electrons inside the target, resulting in a large electrostatic field at the lasertarget interface, which accelerates protons to high energy in the backward direction. While the scaling from protons emitted at the target rear $\left(\sim \mathrm{I}^{0.75}\right)$ is consistent with previously published TNSA models for this interaction regime [17], where an intensity-dependent absorption increase leads to a more effective scaling than the widely accepted TNSA dependence $\sqrt{I}$, the scaling of the ions from the front surface remains close to a $\sqrt{I}$, dependence. We argue that the observed asymmetry and the front-surface scaling can be explained on the basis of our model. We also show that the cut-off energies observed in the experiment are also broadly consistent with the model, although clearly this cannot describe in detail the whole processes which are obviously more complex than our assumptions. Multidimensional simulations and a better characterization of laser-target parameters are needed to get a more refined estimate of the relevant plasma parameters and to elucidate the consequences (if any) of this scenario on proton energies accelerated from the rear surface of the target, e.g., if there is any correlation between rear and front surface acceleration.

This work was supported by the Institute for Basic Science (IBS) under IBS-R012-D1. The authors acknowledge funding from EPSRC, through grants EP/J002550/1, EP/L002221/1, EP/K022415/1, EP/J500094/1 and from Russian Foundation for Basic Research through grants 15-02-03042 and 16-02-00088. 
[2] A. Jullien, O. Albert, F. Burgy, G. Hamoniaux, J.-P. Rousseau, J.-P. Chambaret, F. Augé-Rochereau, G. Chériaux, J. Etchepare, N. Minkovski, and S. M. Saltiel, Opt. Lett. 30, 920 (2005).

[3] C. Thaury, F. Quéré, J.-P. Geindre, A. Levy, T. Ceccotti, P. Monot, M. Bougeard, F. Réau, P. d'Oliveira, P. Audebert, R. Marjoribanks, and Ph. Martin, Nat. Phys. 3, 424 (2007).

[4] J. H. Sung, S. K. Lee, T. M. Jeong, and C. H. Nam, Appl. Phys. B 116, 287 (2014).

[5] S. C. Wilks, A. B. Langdon, T. E. Cowan, M. Roth, M. Singh, S. Hatchett, M. H. Key, D. Pennington, A. MacKinnon, and R. A. Snavely, Phys. Plasmas 8, 542 (2001).

[6] J. Fuchs, P. Antici, E. d'Humières, E. Lefebvre, M. Borghesi, E. Brambrink, C. A. Cecchetti, M. Kaluza, V. Malka, M. Manclossi, S. Meyroneinc, P. Mora, J. Schreiber, T. Toncian, H. Pépin, and P. Audebert, Nat. Phys. 2, 48 (2006).

[7] K. Zeil, S. D. Kraft, S. Bock, M. Bussmann, T. E. Cowan, T. Kluge, J.Metzkes, T. Richter, R. Sauerbrey, and U. Schramm, New. J. Phys, 12, 45015 (2010).

[8] T. Ceccotti, A. Lévy, H. Popescu, F. Réau, P. D’Oliveira, P. Monot, J. P. Geindre, E. Lefebvre, and Ph. Martin, Phys. Rev. Lett. 99, 185002 (2007).

[9] S. Fourmaux, S. Buffechoux, B. Albertazzi, D. Capelli, A. Lévy, S. Gnedyuk, L. Lecherbourg, P. Lassonde, S. Payeur, P. Antici, H. Pépin, R. S. Marjoribanks, J. Fuchs, and J. C. Kieffer, Phys. Plasmas 20, 013110 (2013).

[10]R. Prasad, A. A. Andreev, S. Ter-Avetisyan, D. Doria, K. E. Quinn, L. Romagnani, C. M. Brenner, D. C. Carroll, N. P. Dover, D. Neely, P. S. Foster, P. Gallegos, J. S. Green, P. McKenna, Z. Najmudin, C. A. J. Palmer, J. Schreiber, M. J. V. Streeter, O. Tresca, M. Zepf, and M. Borghesi, Appl. Phys. Lett. 99, 121504 (2011).

[11] M. Passoni, L. Bertagna, and A. Zani, New J. Phys, 12, 045012 (2010).

[12]D. C. Carroll, O. Tresca, R. Prasad, L. Romagnani, P. S. Foster, P. Gallegos, S. Ter-Avetisyan, J. S. Green, M. J. V. Streeter, N. Dover, C.A. J. Palmer, C.M. Brenner, F.H. Cameron, K.E. Quinn, J. Schreiber, A.P.L. Robinson, T. Baeva, M.N. Quinn, X.H. Yuan, Z. Najmudin, M. Zepf, D. Neely, M. Borghesi, and P. McKenna, New J. Phys. 12, 045020 (2010).

[13] V. F. Kovalev, V. Yu. Bychenkov, and V. T. Tikhonchuk, JETP 95, 226 (2002).

[14] P. Mora, Phys. Rev. Lett. 90, 185002 (2003).

[15] T. J. Yu, S. K. Lee, J. H. Sung, J. W. Yoon, T. M. Jeong, and J. Lee, Opt. Express 20, 10807 (2012).

[16]P. K. Singh, K.F. Kakolee, T.W. Jeong, and S. Ter-Avetisyan, Nucl. Instrum. Meth. Phys. Res. A 829, 363 (2016).

[17] T. W. Jeong, P. K. Singh, C. Scullion, H. Ahmed, K. F. Kakolee, P. Hadjisolomou, A. Alejo, S. Kar, M. Borghesi, and S. Ter-Avetisyan, Rev. Sci. Instrum. 87, 083301 (2016).

[18]A. V. Brantov, E.A. Govras, V. Yu. Bychenkov, and W. Rozmus, Phys. Rev. ST Accel. Beams 18, 021301 (2015).

[19] F. Cattani, A. Kim, D. Anderson, and M. Lisak, Phys. Rev. E 62, 1234 (2000).

[20] V. V. Goloviznin, and T. J. Schep, Phys. Plasmas 7, 1564 (2000). 
[22] A. V. Korzhimanov, A. A. Gonoskov, A. V. Kim, and A. M. Sergeev, JETP Lett. 86, 577 (2007).

[23]E. Siminos, M. Grech, S. Skupin, T. Schlegel, and V. T. Tikhonchuk, Phys. Rev. E 86, 056404 (2012).

[24] V. A. Vshivkov, N. M. Naumova, F. Pegoraro, and S. V. Bulanov, Phys. Plasmas 5, 2727 (1998).

[25] W. L. Kruer, and K. Estabrook, Phys. Fluids 28, 430 (1985).

[26]F. Brunel, Phys. Rev. Lett. 59, 52 (1987).

[27] V. Yu. Bychenkov, V. N. Novikov, D. Batani, V. T. Tikhonchuk, and S. G. Bochkarev, Phys. Plasmas 11, 3242 (2004).

[28] S. Ter-Avetisyan, M. Schnuerer, S. Busch, E. Risse, P. V. Nickles, and W. Sandner, Phys. Rev. Lett. 93, 155006 (2004).

[29] J. S. Green, A. P. L. Robinson, N. Booth, D. C. Carroll, R. J. Dance, R. J. Gray, D. A. MacLellan, P. McKenna, C. D. Murphy, D. Rusby, and L. Wilson, Appl. Phys. Lett. 104, 214101 (2014).

[30] Y. Fang, X. Ge, S. Yang, W. Wei, T. Yu, F. Liu, M. Chen, J. Liu, X. Yuan, Z. M. Sheng and J. Zhang, Plasma Phys. Control. Fusion 58, 075010 (2016).

[31] S. Fritzler, V. Malka, G. Grillon, J. P. Rousseau, F. Burgy, E. Lefebvre, E. d'Humières, P. McKenna, and K. W. D. Ledingham, Appl. Phys. Lett. 83, 3039 (2003)

[32] M. Schnürer, A. A. Andreev, S. Steinke, T. Sokollik, T. Paasch-Colberg, P. V. Nickles, A. Henig, D. jung, D. Kiefer, R. Hörlein, J. Schreiber, T. Tajima, D. Habs, and W. Sandner, Laser Part. Beams 29, 437 (2011).

[33]B. Aurand, L. Senje, K. Svensson, M. Hansson, A. Higginson, A. Gonoskov, M. Marklund, A. Persson, O. Lundh, D. Neely, P. McKenna, and C. G. Wahlström, Phys. Plasmas, 23, 023113 (2016).

[34] A. Yogo, H. Daido, S. V. Bulanov, K. Nemoto, Y. Oishi, T. Nayuki, T. Fujii, K. Ogura, S. Orimo, A. Sagisaka, J. L. Ma, T. Zh. Esirkepov, M. Mori, M. Nishiuchi, A. S. Pirozhkov, S. Nakamura, A. Noda, H. Nagatomo, T. Kimura, and T. Tajima, Phys. Rev. E., 77, 016401 (2008).

[35] I. Spencer, K. W. D. Ledingham, P. McKenna, T. McCanny, R. P. Singhal, P. S. Foster, D. Neely, A. J. Langley, E.J. Divall, C. J. Hooker, R.J. Clarke, P. A. Norreys, E.L. Clark, K. Krushelnick, and J. R. Davies, Phys. Rev. E 67, 046402 (2003).

[36] Y. Oishi, T. Nayuki, T. Fujii, Y. Takizawa, X. Wang, T. Yamazaki, K. Nemoto, T. Kayoiji, T. Sekiya, K. Horioka, Y. Okano, Y. Hironaka, K. G. Nakamura, K. Kondo, and A. A. Andreev, Phys. Plasmas, 12, 073102 (2005).

[37]D. Neely, P. Foster, A. Robinson, F. Lindau, O. Lundh, A. Persson, C.-G. Wahlström and P. McKenna, Appl. Phys. Lett. 89, 021502 (2006).

[38] M.H.Xu, Y.T.Li, D.C. Carroll, P.S. Foster, S. Hawkes, S. Kar, F. Liu, K. Markey, P. McKenna, M. J.V. Streeter, C. Spindloe, Z.M. Sheng, C.-G. Wahlström, M. Zepf, J. Zheng, J. Zhang, and D. Neely, Appl. Phys. Lett. 100, 084101 (2012) 
Fig. 1. Sketch of the experimental set up. Laser pulse is focused onto a $A l$ target at $30^{\circ}$ of incidence. The accelerated ions along the rear surface target normal and front surface target normal directions are measured by Thomson parabola spectrometers.

Fig. 2. Raw parabolic traces of ions accelerated along a) and b) rear surface target normal and d) and e) front surface target normal directions at the intensities of $8 \times 10^{20} \mathrm{~W} / \mathrm{cm}^{2}$ for a) and d) and $2.5 \times 10^{20} \mathrm{~W} / \mathrm{cm}^{2}$ for b) and e). Scan of proton energy distribution in c) forward and f) backward directions.

Fig. 3. Intensity dependent proton cut-off energies for $A l$ target with a thickness of $6 \mu \mathrm{m}$ along the target normal a) front and b) rear directions.

Fig.4. The measured maximum proton energies at short laser pulse $(\tau<60 \mathrm{fs})$ interaction with thin foils $(1>0.1$ $\mu \mathrm{m})$, where TNSA-like acceleration scheme is applicable, as a function of laser intensity from the a) rear and b) front side of the target. The published results are taken from the reference shown in square brackets.

Fig. 5. Schematic representation of the laser intensity (blue), electron and ion densities (brown and green, correspondingly) and the electric field (red) near the target front side for a) $Q_{e}^{(s)}=Q_{i}$, and b) $Q_{e}^{(s)}<Q_{i}$ 

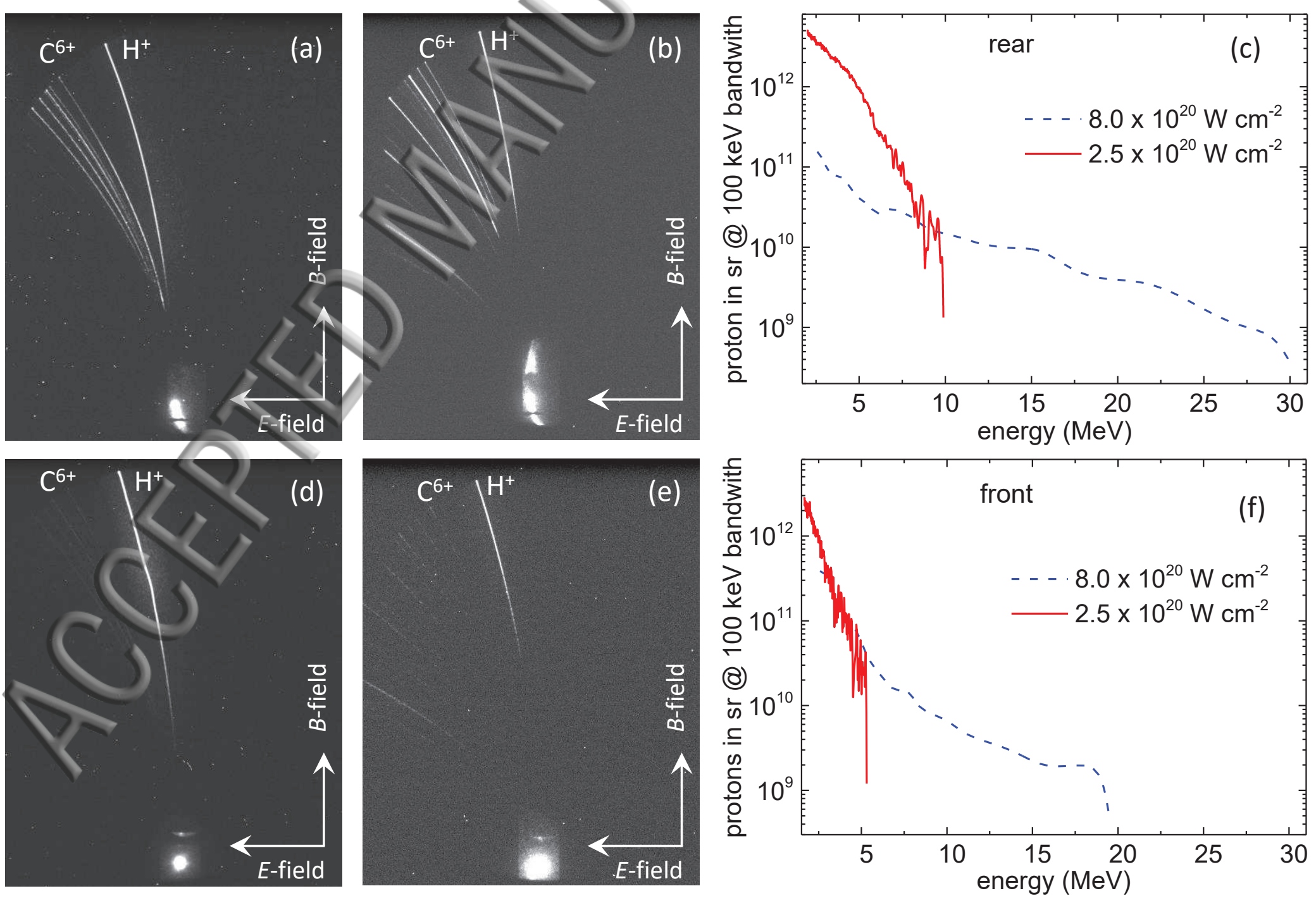

Fig. 2 


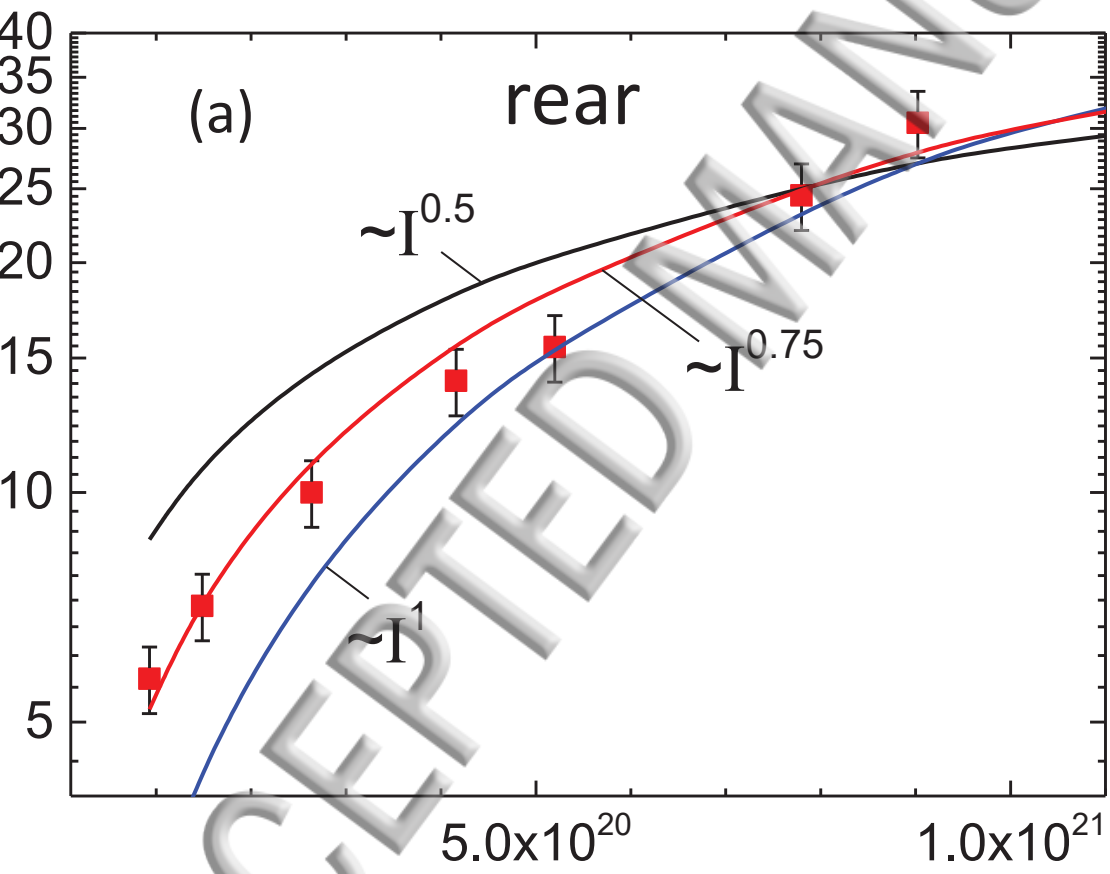

laser intensity $\left(\mathrm{W} / \mathrm{cm}^{2}\right)$

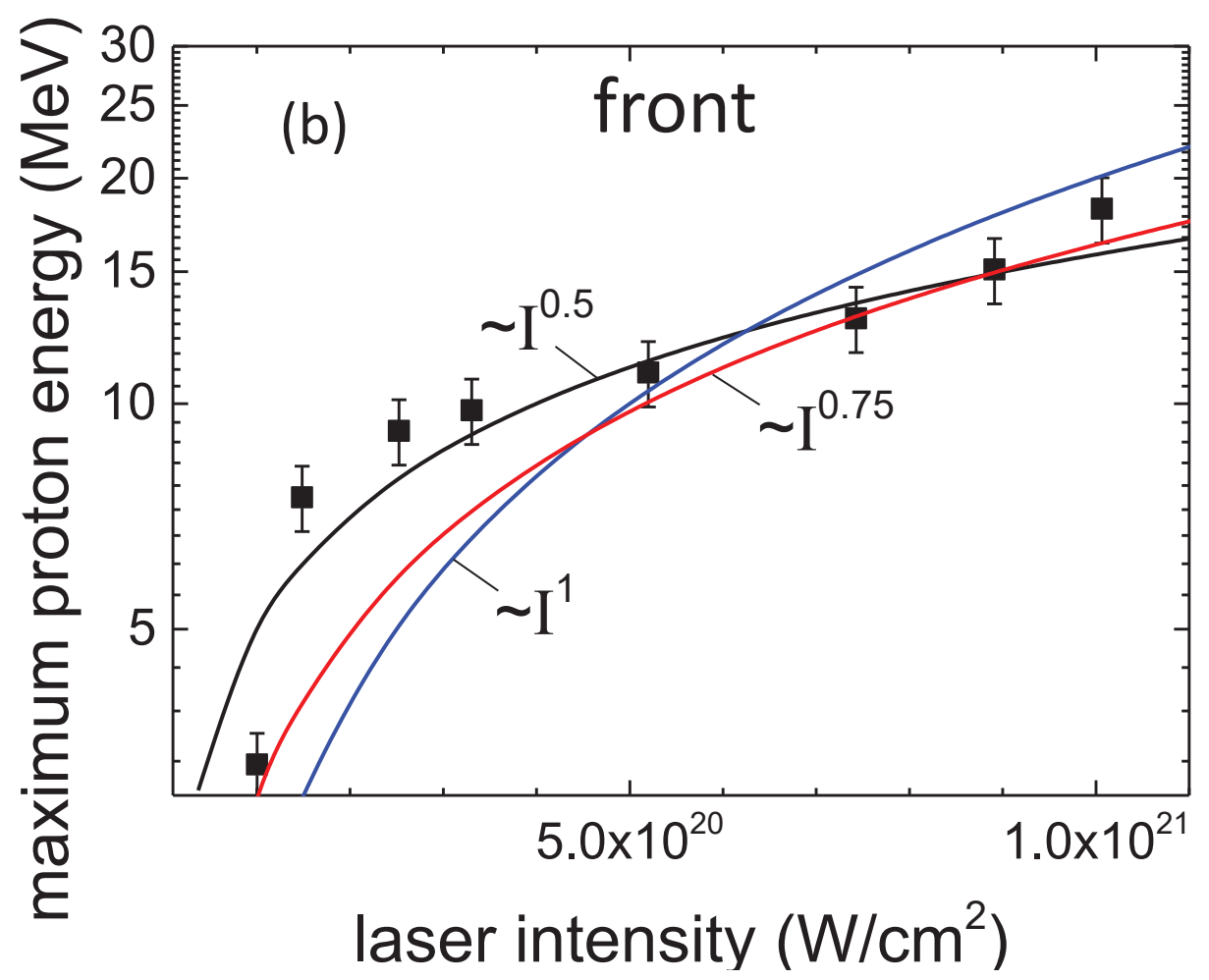

Fig. 3 

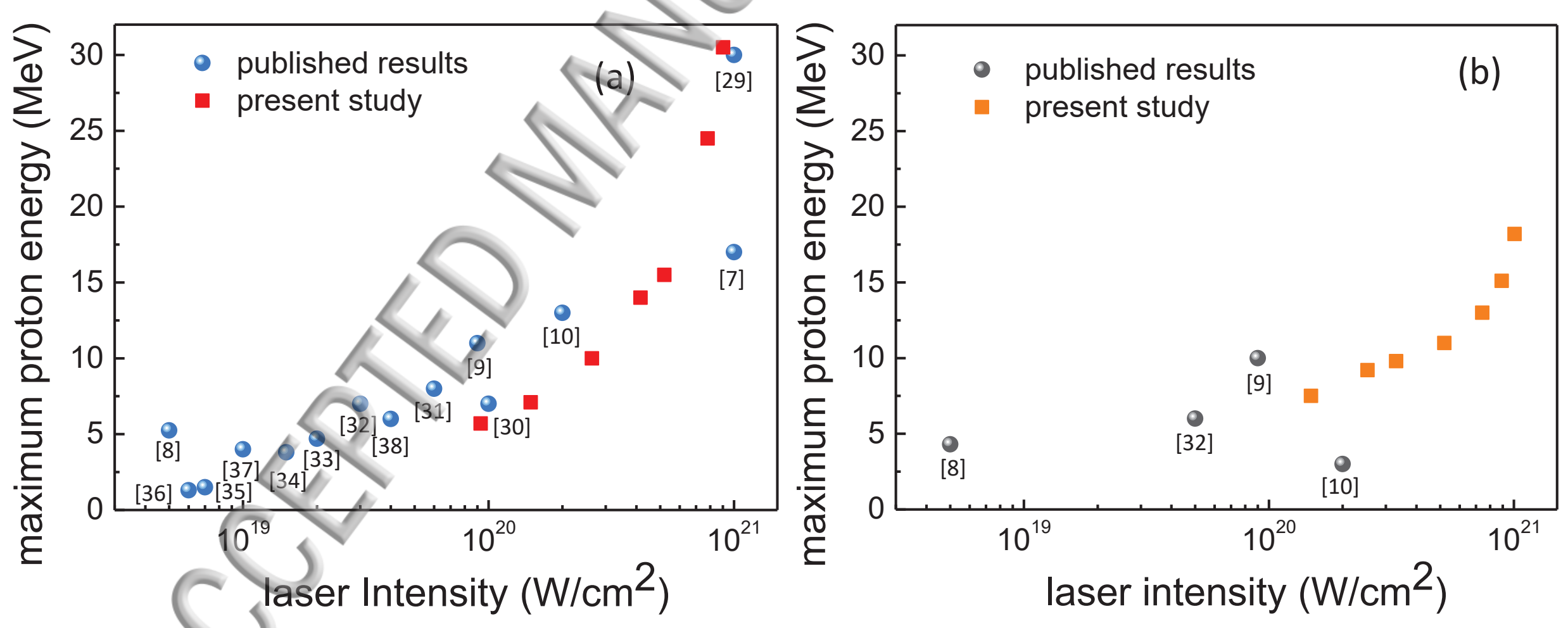

Fig. 4 

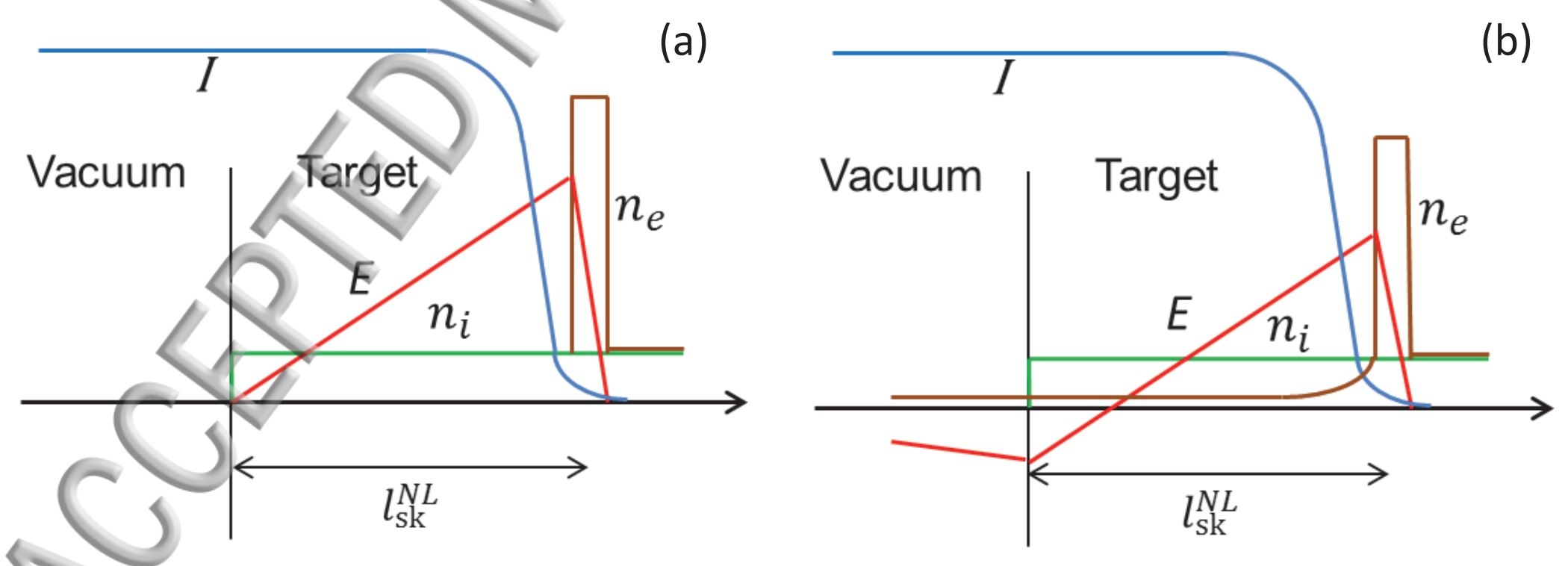

Fig. 5 